\title{
Efeito do fungo Paecilomyces lilacinus sobre ovos de Taenia saginata
}

\author{
Effect of the fungus Paecilomyces lilacinus \\ on Taenia saginata eggs
}

\author{
Fabio Ribeiro Braga ${ }^{1}$, Jackson Victor de Araújoํㅜ, Juliana Milani Araujo ${ }^{1}$, \\ Rogério Oliva Carvalho ${ }^{1}$ e André Ricardo Silva ${ }^{1}$
}

\begin{abstract}
RESUMO
Com o objetivo de demonstrar a eficácia do fungo Paecilomyces lilacinus sobre ovos de Taenia saginata em condições laboratoriais, foi montado ensaio em placas de Petri com agar - água $2 \%$. Houve atividade ovicida $(\mathrm{p}<0,05)$ em relação ao grupo controle no décimo dia de interação e colonização interna dos ovos de $25,5 \%$.
\end{abstract}

Palavras-chaves: Fungos nematófagos. Paecylomices lilacinus. Taenia saginata. Controle biológico.

\begin{abstract}
With the aim of demonstrating the effectiveness of the fungus Paecilomyces lilacinus on Taenia saginata eggs under laboratory conditions, a trial was set up in Petri dishes with water-agar $2 \%$. There was ovicidal activity $(\mathrm{p}<0.05)$ in relation to the control group on the tenth day of interaction and an internal colonization rate of $25.5 \%$ in the eggs.
\end{abstract}

Key-words: Nematophagous fungi. Paecylomices lilacinus. Taenia saginata. Biological control.

O complexo teníase/cisticercose por Taenia saginata possui distribuição cosmopolita e está amplamente difundido na maioria dos países onde existe a atividade pecuária bovina. Todavia, o Brasil possui uma situação privilegiada no cenário mundial na criação de bovinos, pois se apresenta como o maior produtor de rebanho comercial ${ }^{2}$.

O ciclo da Taenia saginata implica dois hospedeiros, um definitivo e um intermediário, e uma fase de vida livre. 0 único hospedeiro definitivo de ambas as tênias (fase adulta do parasita) é o ser humano, em cujo intestino delgado se alojam. Por sua vez, o hospedeiro intermediário de Taenia saginata são os bovinos, desenvolvendo-se na sua musculatura. Três fases em relação à população desses parasitas são reconhecidas: adulto no hospedeiro definitivo, ovos no ambiente e a fase larval no hospedeiro intermediário ${ }^{1}$. Segundo Gemmell \& Lawson ${ }^{9}$ alguns fatores podem contribuir de forma favorável para a dispersão dos ovos de Taenia saginata no ambiente e, dentre esses está à contaminação fecal do solo. 0 ser humano pode se contaminar através da ingestão de carne crua ou mal cozida contendo cisticercos e a doença, teníase, pode se apresentar de forma assintomática, porém em alguns casos podem coexistir alterações no apetite, náuseas, vômitos, dores abdominais, diarréias, emagrecimento, irritabilidade e fadiga ${ }^{615}$.

No combate ao complexo teníase/cisticercose, várias medidas podem ser empregadas, mas a principal estratégia consiste na interrupção do ciclo evolutivo do parasita ${ }^{13}$. Porém, medidas alternativas que possam ser empregadas no combate à disseminação ambiental, deste, e de outros parasitas gastrintestinais, potencialmente zoonóticos, e suas formas infectantes, são bem vindas. Entre elas está o uso de fungos nematófagos que são antagonistas naturais de helmintos ${ }^{3}$. Algumas espécies de fungos podem ser utilizadas como potencias agentes de controle de helmintoses originadas a partir do ambiente ${ }^{7}$. Dentre esses fungos destacam-se as espécies Paecilomyces lilacinus e Pochonia chlamydosporia consideradas ovicidas, pois apresentam colonização interna dos ovos parasitados e por conseqüência seu rompimento ${ }^{8510}$.

Esse estudo procurou demonstrar o efeito ovicida do fungo Paecilomyces lilacinus sobre ovos de Taenia saginata.

0 fungo nematófago Paecilomyces lilacinus foi mantido em tubos de ensaio a $4^{\circ} \mathrm{C}$ contendo corn-meal-ágar $2 \%$ e no escuro durante 10 dias.

\footnotetext{
1. Departamento de Veterinária, Universidade Federal de Viçosa, Viçosa, MG. Apoio financeiro: CNPq.

Endereço para correspondência: Dr. Fabio R. Braga. Dept ${ }^{0}$ de Veterinária/ UFV. Av. Ph Rolfes s/n, 36570-000 Viçosa, MG.

Tel: $55313899-1458$.

e-mail: fabioribeirobraga@hotmail.com

Recebido para publicação em 21/01/2008

Aceito em 09/10/2008
} 
Após o crescimento, novos discos de cultura de $4 \mathrm{~mm}$ de diâmetro foram transferidos para placas de Petri de $9 \mathrm{~cm}$ diâmetro contendo 20mL de ágar-água 2\% durante 10 dias. Os ovos de Taenia saginata foram recuperados a partir da dissecação de proglotes de um exemplar adulto e, analisados morfologicamente quanto a sua integridade ao microscópio óptico em objetiva de 10x.

Os ovos foram vertidos sobre a superfície de placas de Petri de $9 \mathrm{~cm}$ de diâmetro contendo o meio (AA 2\%) com o isolado fúngico crescido por 10 dias e sem fungo como controle, sendo feitas 10 repetições para cada grupo. Nos tratamentos, cada placa continha mil ovos de Taenia saginata com apenas o fungo. Ao final de 10 dias, aproximadamente cem ovos foram retirados de cada placa contendo o fungo e do controle (sem fungo) de acordo com a técnica descrita por Araújo e cols ${ }^{4}$ sendo então avaliados em objetiva de 40x de acordo com os parâmetros estabelecidos por Lysek e cols ${ }^{11}$ : tipo 1, efeito lítico sem prejuízo morfológico à casca do ovo, onde hifas são observadas aderidas à casca; tipo 2, efeito lítico com alteração morfológica da casca e embrião do ovo, sem penetração de hifas através da casca e tipo 3, efeito lítico com alteração morfológica do embrião e da casca, além de penetração de hifas e colonização interna do ovo. Os dados do intervalo estudado foram submetidos ao teste não paramétrico de Friedman com 5\% de probabilidade.

Os resultados percentuais para os efeitos dos tipos 1, 2 e 3 aos 10 dias do fungo Paecilomyces lilacinus estão representados na Figura 1. A análise dos dados revelou diferença $(\mathrm{P}<0,05)$ para os efeitos dos tipos 1, 2 e 3 apresentados por Paecilomyces lilacinus em relação ao grupo controle. Com a microscopia óptica em objetiva de 40x, foram observados hifas desse fungo no interior dos ovos de Taenia saginata proporcionando seu rompimento (Figura 2), e com isso caracterizando o efeito do tipo $3^{10}$.

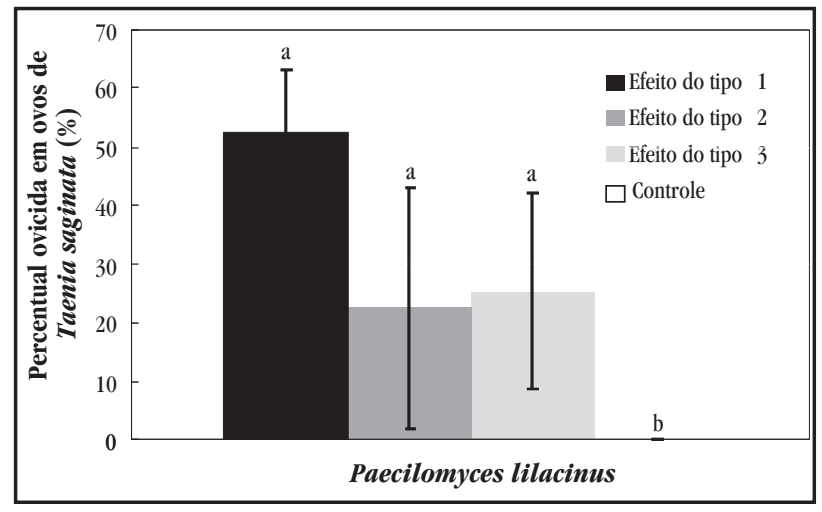

Figura 1 - Percentuais e desvio padrão (barra) para os efeitos dos tipos $1^{*}, 2^{* *}$ e $3^{* * *}$ do fungo nematófago Paecilomyces lilacinus sobre ovos de Taenia saginata e grupo controle aos 10 dias de interação.

Percentual seguido de letra igual maiúscula não difere estatisticamente $(\mathrm{P}>0,05)$ - teste de Friedman.

*efeito lítico sem prejuízo morfológico à casca do ovo, onde as hifas são observadas aderidas à casca.

**efeito lítico com alteração morfológica da casca do ovo e do embrião, sem penetração de hifas através da casca.

****efeito lítico com alteração morfológica da casca e do embrião, além da penetração de hifas e colonização interna do ovo.

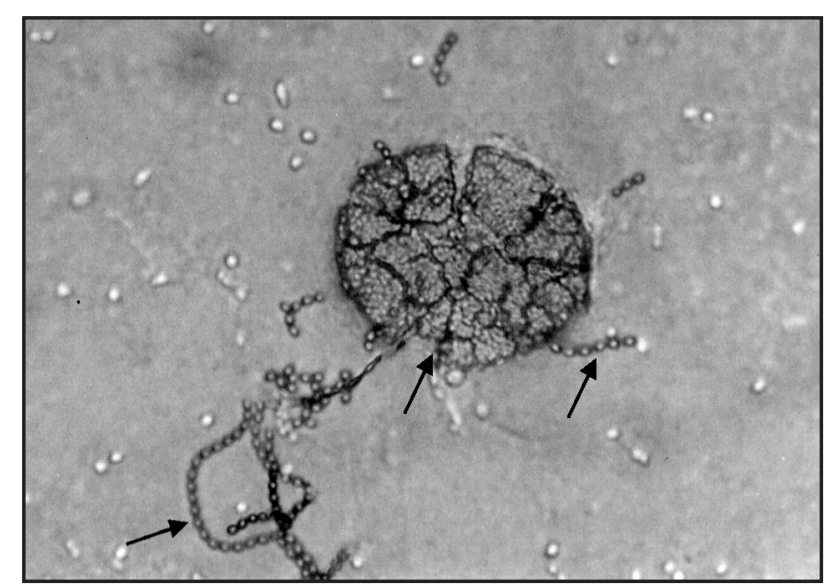

Figura 2 - Hifas do fungo Paecilomyces lilacinus (seta branca) e ovo rompido de Taenia saginata (seta preta) aos 10 dias de interação. Microscopia óptica c/ aumento de 40x.

As espécies de fungos ovicidas têm sido utilizadas com sucesso no controle in vitro de ovos de helmintos. Braga e cols ${ }^{5}$ demonstram a eficácia de dois isolados do fungo Pochonia chlamydosporia sobre ovos de Fasciola hepatica nos intervalos de sete, 14 e 21 dias, encontrando percentuais para a atividade ovicida, efeito do tipo 3 , acima de $20 \%$ ao final do experimento para ambos os isolados testados. Em outro trabalho, Araújo e cols ${ }^{4}$ demonstraram a ação de Paecilomyces lilacinus sobre ovos de Toxocara canis registrando resultados percentuais ao final de sete dias para o efeito do tipo 3 de 16\% e, para os efeitos dos tipos 1 e 2 esses autores registraram 45\% e 15\%, respectivamente.

Comparando os resultados obtidos no presente trabalho com os resultados registrados por Araújo e cols ${ }^{4}$, nota-se semelhança no modo de ação desse fungo sobre ovos de Taenia saginata, uma vez que, houve apresentação do efeito do tipo 3 de $25,5 \%$ e o maior valor percentual observado foi para o efeito do tipo 1 de 52,5\%. Segundo Lysek ${ }^{10}$ um fungo é considerado ovicida se apresentar sobre os ovos parasitados o efeito do tipo 3, fato este, que ocorreu em ambos os trabalhos. Contudo, O'Hara \& Jatala ${ }^{13}$ mencionam que a frequiência e o sucesso da penetração em ovos por fungos são aparentemente dependentes da composição da cutícula do ovo. Mas, no caso específico de ovos de Taenia saginata, estes, são bem resistentes, pois possuem uma casca protetora chamada de embrióforo, e podem sobreviver no ambiente por até 12 meses ${ }^{16}$. Dessa maneira, podem-se buscar medidas alternativas que possam auxiliar no combate à disseminação ambiental, deste, e de outros parasitas gastrintestinais, através do controle biológico realizado com fungos nematófagos ovicidas ${ }^{12}$.

Esses resultados demonstram que o fungo Paecilomyces lilacinus apresentou eficácia in vitro sobre ovos de Taenia saginata em condições laboratoriais ao final de dez dias.

\section{REFERÊNCIAS}

1. Acha P, Szifre B. Zoonosis y enfermidades trasmissible comunes al hombre y a los animales. p. 989, 1986.

2. Alves DA. As dificuldades na inspeção de frigoríficos brasileiros no mercado internacional: um estudo sobre a comercialização da carne bovina in natura. Revista Nacional da Carne 25:96-114, 2001. 
3. Araújo JV, Mota MA, Campos AK. Controle biológico de helmintos parasitos de animais por fungos nematófagos. Revista Brasileira de Parasitologia Veterinária 13:165-170, 2004.

4. Araújo JV, Santos MA, Ferraz S. Efeito ovicida de fungos nematófagos sobre ovos embrionados de Toxocara canis. Arquivo Brasileiro de Medicina Veterinária e Zootecnia 47:37-42, 1995

5. Braga FR, Araújo JV, Campos AK, Araújo JM, Silva AR, Carvalho RO, Tavela AO. In vitro evaluation of the action of the nematophagous fungi Duddingtonia flagrans, Monacrosporium sinense and Pochonia chlamydosporia on Fasciola hepatica eggs. World Journal of Microbiology and Biotechnology 24:1559-1564, 2008.

6. Carrada-Bravo T. Teniasis-cisticecosis como problema de salud pública. Boletín Médico del Hospital Infantil del México 44:427-434, 1987.

7. Despommier D. Toxocariasis: clinical aspects, epidemiology, medical ecology, and molecular aspects. Clinical Microbiology Reviews 16:265-272, 2003.

8. Fieldler ZA, Sosnowska D. Nematophagous fungus, Paecilomyces lilacinus (Thom) Samson is also a biological agent for control of greenhouse insects and mite pests. Biological Control 52:547-558, 2007.
9. Gemmell MA, Lawson JR Ovine cysticercosis: an epidemiological model for the cysticercosis. I. Free-living egg fase. In: Flisser A (ed) Cysticercosis: present stage of knowledge and perspectives. New York, p. 87-98, 1982.

10. Lysek $\mathrm{H}$. Classification of ovicide fungi according to type of ovicidity. Acta University Palack Olomue 76:9-13, 1976.

11. Lysek H, Fassatiová O, Pineda NC, Hernández NL. Ovicidal fungi in soils of Cuba. Folia Parasitologica 29:265-270, 1982.

12. Mota MA, Campos AK, Araújo JV. Controle biológico de helmintos parasitos de animais: estágio atual e perspectivas futuras. Pesquisa Veterinária Brasileira 23:93-100, 2003

13. O'Hara CM, Jatala P. Ultraestructure of Meloydogine, Globodera and Nacobus eggs shells as related to the activities of biocontrol fungi. Journal of Nematology 17:508, 1985.

14. Organización Panamericana de La Salud. Epidemiologia y control de la teniasis/ cisticercosis en America Latina. Washington, p. 297, 1994

15. Rey L. Bases da Parasitologia Médica. Rio de Janerio, p. 496-509, 2001

16. Silva AVM. Teníase e Cisticercose. In: Neves DP, Melo AL, Linardi PM (eds) Parasitologia Humana. Editora Atheneu, Rio de Janeiro, p. 227-237, 2005. 